\title{
Flatness Based Control of an Industrial Robot Joint Using Secondary Encoders
}

\author{
Jonas Weigand ${ }^{a, *}$, Nigora Gafur ${ }^{a}$ and Martin Ruskowski ${ }^{a, b}$ \\ ${ }^{a}$ Chair of Machine Tools and Control Systems (WSKL), TU Kaiserslautern, Gottlieb-Daimler-Str. 42, 67663 Kaiserslautern, Germany \\ ${ }^{b}$ Innovative Factory Systems (IFS), German Research Center for Artificial Intelligence (DFKI), Trippstadter Str. 122, 67663 Kaiserslautern, Germany
}

\section{ARTICLE INFO}

\section{Keywords:}

industrial robot

flexible joint model

flatness based feed forward control

robot machining

secondary encoders

computed torque control

\begin{abstract}
A B S T R ACT
Due to their compliant structure, industrial robots without precision-enhancing measures are only to a limited extent suitable for machining applications. Apart from structural, thermal and bearing deformations, the main cause for compliant structure is backlash of transmission drives. This paper proposes a method to improve trajectory tracking accuracy by using secondary encoders and applying a feedback and a flatness based feed forward control strategy. For this purpose, a novel nonlinear, continuously differentiable dynamical model of a flexible robot joint is presented. The robot joint is modeled as a two-mass oscillator with pose-dependent inertia, nonlinear friction and nonlinear stiffness, including backlash. A flatness based feed forward control is designed to improve the guiding behaviour and a feedback controller, based on secondary encoders, is implemented for disturbance compensation. Using Automatic Differentiation, the nonlinear feed forward controller can be computed in a few microseconds online. Finally, the proposed algorithms are evaluated in simulations and experimentally on a real KUKA Quantec KR300 Ultra SE.
\end{abstract}

\section{Introduction}

Industrial robots are highly flexible due to their open and complex kinematic chain and are mainly designed for a good repeatability but not for high precision tasks, such as milling [1]. The main drawback of using an industrial robot in machining processes is the lack of high position accuracy caused by low stiffness, meaning low eigenfrequencies and joint elasticity between the actuators and the driven links $[2,3]$. As a result, conventional computerized numerical control (CNC) machines, possessing high stiffness and simple kinematics, can still not be replaced by industrial robots for machining tasks $[4,5]$. The low and highly pose dependent overall stiffness of industrial robots, which is up to 100 times less than of conventional CNC machines, can lead to vibrations and chattering effects, so that the machining quality suffers [6-8]. Already the influence of the placement of a workpiece with respect to the robot can lead to different machining results due to pose dependent stiffness of industrial robots [9]. Two different methods to increase the machining accuracy of industrial robots are reported in the literature, either to use a model-based approach accounting for compliance of flexible joints or to use a sensor-based approach by tracking deformations $[1,3,10,11]$. The sensor-based approach can lead to a higher position accuracy than a modelbased approach [3]. Attaching secondary encoders (SE) to the link-side can reduce the oscillatory behaviour and chattering effects by providing sensor information to a feedback controller. An improvement of static precision by a factor of 10 was achieved in [12] using SE. However, the approach is associated with high costs, high implementation effort, communication delays and sensor noise [3]. Despite of that, re-

@jonas.weigand@mv.uni-kl.de (J. Weigand)

ORCID(s): 0000-0001-5835-3106 (J. Weigand); 0000-0002-3131-3574 (N. Gafur); 0000-0002-6534-9057 (M. Ruskowski) ducing dynamic deflections, due to time-varying dynamical effects, still remains a challenge that can only be handled by a model-based approach.

Modeling joint flexibility involves considering stiffness and friction between motor and the driven link, as well as effects occurring in transmission drives, such as backlash, lost-motion and hysteresis effects. High precision transmission drives, such as cycloid gears and harmonic drives, are typically used for industrial robots in order to meet high precision requirements. Simplified models taking into account linear stiffness and damping between the motor and the driven link are used in [13-17]. An overview of further simplified models can be found in [18]. Nonlinear modeling approaches account for torsional compliance by considering hysteresis effects $[19,20]$. Hysteresis behavior results from the structural damping of transmission elements and their piecewise elasto-plastic properties [19]. It was shown that hysteresis effects can significantly contribute to a low path accuracy [20]. The authors point out, that it mostly impacts the positional error of the base joint due to the large lever arm. Moreover, hysteresis behavior, which has a bidirectional behaviour, leads to alternating stresses in the gears [21]. The non-linear characteristics of lost-motion and backlash in transmission drives have further considerable effects on lower path accuracy in machining tasks. Lost-motion is defined as the torsion angle at the midpoint of hysteresis curve, where not all tooth-flanks of the gearbox are in full contact, whereas backlash is defined as the angle difference in the output shaft at zero output torque, where gear teeth are not in contact $[22,23]$. The literature on modeling lost-motion effects is scarce. The impact of backlash caused by transmission drives was recognized by [24] in his experimental study of harmonic drive, showing that torque transmission has a nonlinear characteristics and the input torque can not be entirely transmitted to the driven link. The impact 
of backlash is also investigated and modeled in $[2,25]$. The authors in [23] show a considerable improvement in milling accuracy of aluminium by taking into account backlash effects and all joint flexibilities. Moreover, it was shown in [26] that backlash leads to the accumulation of positioning errors while joints change their rotation direction, leading to torque oscillations and thus to earlier gear system failure.

Enhancing trajectory tracking accuracy requires not only considering the most relevant dynamic effects but also designing a proper control law. Chattering effects, which significantly influence the surface quality in a milling process, can only be eliminated using a flexible model-based controller [27]. For the flexible model-based controller, the trajectory should be continuously differentiable up to the $4^{\text {th }}$ order, i.e. up to jerk derivative, whereas a $2^{\text {nd }}$ order trajectory is sufficient for rigid model-based controllers. A continuously differentiable trajectory can be computed e.g. based on the dynamic model or estimated by numerical differentiation, which is error prone due to high sampling frequencies, measurement noise or model uncertainties. The authors in [28] propose a robust adaptive control method for trajectory tracking and an online parameter estimation of a 6 degrees of freedom (DoF) industrial robot. The proposed controller differs from other controllers in the literature, as it is designed in the task space of the robot's end effector in order to achieve better trajectory tracking accuracy compared to controllers designed in joint space. The method shows a significant reduction of trajectory tracking error compared to a conventional PD controller. Similarly, the authors in [17] show an improvement in dynamic path accuracy of a robot manipulator in a machining process, proposing a controller built on an independent joint control. A damping control algorithm is designed and validated experimentally, based on a velocity feedback using SE. The approach is extended by a state estimation. With the proposed controller, it is possible to alter the stiffness and damping of the controller system systematically with help of two proportional gains. The authors in [29] show that modeling nonlinear effects, such as friction, and subsequent derivation of a model-based controller, can lead to a significant improvement in position and velocity accuracy of an industrial robot.

Providing a model which takes nonlinear effects into consideration is critical to trajectory tracking accuracy for manipulator systems. The aim of this paper is to improve trajectory tracking performance of an industrial robot by combining the model-based and sensor-based approaches. A novel nonlinear but continuous differentiable modeling approach for friction, backlash and lost-motion is introduced. This allows a derivation of a flatness based feed forward control law. The feed forward control law, which is mostly preferred as it shows more robustness against sampling rate, sensor noise and parameter uncertainty [27] reduces positional errors up to $60 \%$ [10]. In addition, model errors are compensated by applying a feedback law using SE. We propose an enhanced motor-side velocity controller, which takes flexible joint model into account.

The paper is structured as follows. At first, a nonlin-

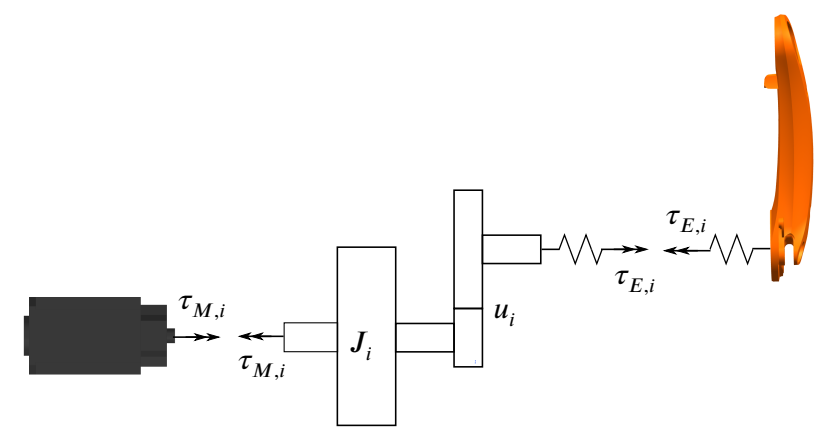

Figure 1: Representation of a flexible robot joint.

ear dynamic model is introduced, followed by Section 3, explaining the advanced control design with feed forward and feedback controller. Further, simulation results are presented and discussed in Section 4, as well as experimental results in Section 5. The paper is finalized by concluding aspects for future research in Section 6.

\section{Modeling}

For modeling a joint flexibility, each motor is considered as a rigid body connected to a driven link via a transmission device and a rotational spring, illustrated in Fig. 1. Such a system can be modeled as a two mass oscillator leading to two generalized coordinates for each single joint. The generalized coordinates are composed of the motor positions $\boldsymbol{\theta} \in \mathbb{R}^{N}$ and the joint positions $\mathbf{q} \in \mathbb{R}^{N}$, where $N$ stands for the number of DoF. That leads to an $2 N$ DoF system of a robot, meaning a 12 DoF system for robot kinematics with 6 joints. The dynamical model of the considered robot manipulator is derived by means of Lagrange's equations of the second kind. The transmission ratios are assumed to be high enough, so that inertial couplings in the acceleration between the motors and the links can be neglected [30]. The governing equations are

$$
\begin{aligned}
\mathbf{J} \ddot{\theta}+\mathbf{U}^{-1} \tau_{E}(\boldsymbol{\theta}, \mathbf{q}) & =\tau_{M}, \\
\mathbf{M}(\mathbf{q}) \ddot{\mathbf{q}}+\mathbf{C}(\mathbf{q}, \dot{\mathbf{q}}) \dot{\mathbf{q}}+\mathbf{g}(\mathbf{q})+\tau_{F}(\dot{\mathbf{q}}) & =\tau_{E}(\boldsymbol{\theta}, \mathbf{q}),
\end{aligned}
$$

where (1) describes the dynamical model of the flexible joints and (2) represents the dynamics of the links.

The two dynamical systems are coupled by the generalized elastic torque vector $\boldsymbol{\tau}_{\boldsymbol{E}}(\boldsymbol{\theta}, \mathbf{q}) \in \mathbb{R}^{N} . \mathbf{J}=\operatorname{diag}\left(\boldsymbol{J}_{1}, \ldots, \boldsymbol{J}_{N}\right)$ is the inertia matrix of the rotors, the vector of generalized friction torques induced by dissipative forces is represented by the vector $\tau_{F}(\dot{\mathbf{q}}) \in \mathbb{R}^{N}$, the input torque transmitted from motors to the driven links is summarized in the generalized vector $\tau_{\boldsymbol{M}} \in \mathbb{R}^{N}$, the transmission ratio for each joint is summarized in the matrix $\mathbf{U}=\operatorname{diag}\left(u_{1}, \ldots, u_{N}\right)$. From (2), the pose dependent inertia matrix of the links is denoted by $\mathbf{M} \in \mathbb{R}^{N \times N}, \mathbf{C}(\mathbf{q}, \dot{\mathbf{q}}) \in \mathbb{R}^{N \times N}$ represents the matrix, which coefficients encompass the centrifugal (proportional to $\dot{q}_{i}^{2}$ ) and Coriolis (proportional to $\dot{q}_{i} \dot{q}_{j}, i \neq j$ ) forces and 


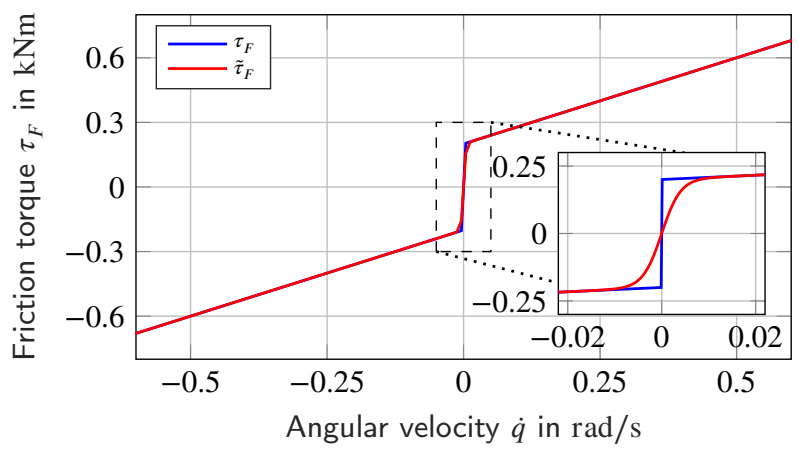

Figure 2: Coulomb and viscous friction model with discontinuous and continuous approximation, exemplary for joint 1. Model parameters are given in Tab. 2.

$\mathbf{g}(\mathbf{q}) \in \mathbb{R}^{N}$ is the vector of gravitational torques. For the sake of simplicity, a single joint will be considered in the following, omitting the indices of the variables indicating a specific joint e.g. $v_{i}$ is the $i$-th element of the vector $\mathbf{v} \in \mathbb{R}^{N}$, simplified to $v_{i}=v$.

\subsection{Friction Model}

The gearbox friction is modeled as Coulomb and viscous friction. The temperature dependency of the friction is neglected in this paper. With the viscous friction constant $f_{v}$ and the Coulomb friction torque $f_{c}$ we get

$$
\tau_{F}= \begin{cases}0, & \dot{q}=0 \\ f_{v} \dot{q}+f_{c} \operatorname{sign}(\dot{q}), & \text { otherwise. }\end{cases}
$$

For the implementation of a flatness based controller, a continuously differentiable function is needed. Therefore, an approximation of the friction is introduced, $\tilde{\tau}_{F} \approx \tau_{F}$, using an exponential function and a friction smoothness factor $s_{F}$

$$
\tilde{\tau}_{F}(\dot{q})=f_{v} \dot{q}+\frac{2 f_{c}}{1+e^{-s_{F}} \dot{q}}-f_{c} .
$$

The functions (3) and (4) are illustrated in Fig. 2.

\subsection{Stiffness Model}

The stiffness model considers backlash, lost-motion, and linear elasticity. Lost-motion describes an effect in between backlash and linear elasticity, where not all tooth flanks are in full contact. During backlash the elastic torque vanishes, i.e. $\tau_{E}=0$. In the lost-motion range, the stiffness is modeled as linear with a smaller slope and an offset. In the torsional rigidity range, the stiffness is modeled as linear with an offset, as shown in Fig. 3. We define the stiffness coefficients $c_{L M}$ and $c_{T R}$, for lost-motion and torsional rigidity respectively. We further define the angular ranges $\phi_{B *}$ and $\phi_{L M}$, for backlash and lost-motion respectively. The torsional ridigity is defined as a measurement between $50 \%$ and $100 \%$ of the nominal torque. Lost-motion effects occur directly after the backlash and are measured between $\pm 3 \%$ of

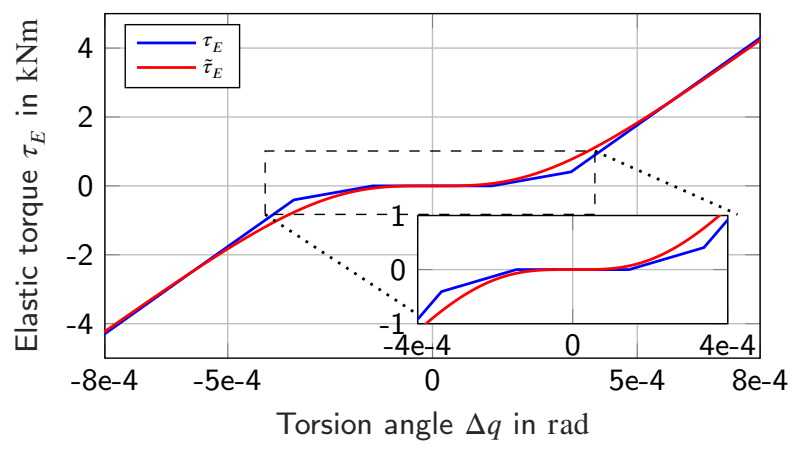

Figure 3: Discontinuous and continuous approximation for stiffness model with the effects of backlash, lost-motion and linear elasticity, exemplary for joint 1 . Model parameters are given in Tab. 2.

the nominal torque [22]. Therefore, we introduce an effective backlash angle $\phi_{B}=\phi_{B *}+\phi_{L M}$ which ensures a correct modeling of the lost-motion and torsional rigidity range. With the link torsion angle and its sign function

$$
\begin{aligned}
\Delta q & =\theta / u-q, \\
\sigma & =\operatorname{sign}(\Delta q)
\end{aligned}
$$

and the elastic torque offset $\tau_{E, 0}=c_{L M} \phi_{L M}$, the elastic torque can be obtained

$$
\tau_{E}= \begin{cases}0, & |\Delta q| \leq \phi_{B *} \\ c_{L M}\left(\Delta q-\frac{\phi_{L M} \sigma}{2}\right), & \phi_{B *}<|\Delta q| \leq \phi_{B} \\ c_{T R} \Delta q+\tau_{E, 0} \sigma, & \text { otherwise }\end{cases}
$$

Although (6) describes the nonlinear stiffness precisely, it is difficult to use it in parameter estimation or advanced control strategies, such as Model Predictive Control (MPC), since the function is not continuously differentiable. Problems with (6) potentially arise with all derivative based algorithms. In particular, many Automatic Differentiation (AD) tools, such as implemented in the Symbolic Math Toolbox from $M A T L A B$ [31] require continuously differentiable equations. As a main contribution of this paper, we present a continuously differentiable elastic torque function, which origins from time-domain considerations as explained in Appendix B and is applied in the following. To derive a differentiable approximation $\tilde{\tau}_{E} \approx \tau_{E}$ of (6), we define

$$
\begin{aligned}
\tilde{\tau}_{E,+}(\Delta q) & =c_{T R} \Delta q-c_{T R} \phi_{B} \\
& +c_{T R} \phi_{B} e^{-\left(3 \Delta q / \phi_{B}\right)} \\
& +2 c_{T R} \Delta q e^{-\left(3 \Delta q / \phi_{B}\right)} \\
& +3 c_{T R} /\left(2 \phi_{B}\right)(\Delta q)^{2} e^{-\left(3 \Delta q / \phi_{B}\right)}
\end{aligned}
$$

for all $\Delta q \geq 0$ and

$$
\tilde{\tau}_{E,-}(\Delta q)=-\tilde{\tau}_{E,+}(-\Delta q)
$$




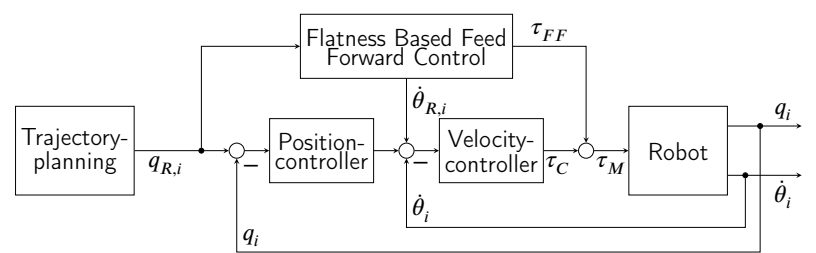

Figure 4: Controller design with feed forward and feedback control

for all $\Delta q<0$. A complete continuously differentiable elastic torque function is given by

$$
\tilde{\tau}_{E}=\tanh \left(s_{E 1} \Delta q\right) \tilde{\tau}_{E,+}\left(\Delta q \cdot \tanh \left(s_{E 1} \Delta q\right)\right)
$$

for all $\Delta q \in \mathbb{R}$ with the hyperbolic tangent $\tanh (\cdot)$. It is essential for the tangent slope factor $s_{E 1}$ that $s_{E 1} \gg 3 / \phi_{B}$ holds. Note that the slope factor is not upper-bounded, except for limits due to numerical considerations. The continuously differentiable stiffness curve is depicted in Fig. 3.

For many model applications, such as MPC or parameter identification, a continuously differentiable stiffness function is beneficial. However, a flatness based control is an exception, where both, (9) and the inverse stiffness function (10) can be employed. Although the inverse stiffness function is neither common nor applicable in general, it is advantageous in the case of flatness based control to use the following form

$$
\Delta q= \begin{cases}0, & \tau_{E}=0 \\ \frac{\tau_{E}}{c_{L M}}+\frac{\phi_{B *}}{2} \operatorname{sign}\left(\tau_{E}\right), & 0<\left|\tau_{E}\right| \leq \tau_{E, 0} \\ \frac{\tau_{E}}{c_{T R}}+\phi_{B} \operatorname{sign}\left(\tau_{E}\right), & \tau_{E, 0}<\left|\tau_{E}\right|\end{cases}
$$

since the inverse stiffness function reduces the number of required exponential functions from eight in (9) to one in (11). For (10) we can apply the same method as for the nonlinear friction, and we obtain the nonlinear, inverse, continuously differentiable stiffness function

$$
\Delta q=\frac{\tau_{E}}{c_{T R}}+\frac{2 \phi_{B}}{1+e^{-s_{E 2} \tau_{E}}}-\phi_{B}
$$

for all $\Delta q \in \mathbb{R}$ with the elastic smoothing factor $s_{E 2}$.

\section{Controller Design}

We propose a model-based feed forward controller to account for the dynamical system and a feedback control law for compensation of unknown disturbances and model errors. In Fig. 4 and Fig. 5 two different control architectures are presented. Both of them contain a feed forward controller, a feedback controller and trajectory planning.

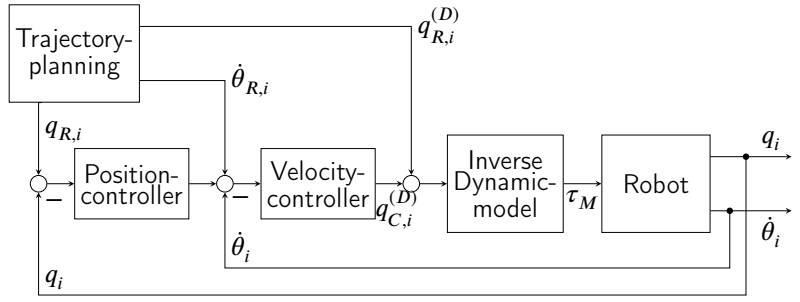

Figure 5: Controller design with exact linearization

The trajectory planning generates the reference signals, i.e. the link angle $q_{R}$ and its derivatives of a desired trajectory. A motor torque $\tau_{M}$ is applied to the robot and the link angle $q$ and the motor velocity $\dot{\theta}$ are the control variables of the feedback controller. The link angle $q$ can be measured directly using SE and the motor velocity $\dot{\theta}$ can be measured using the motor resolver. For both sensors, noise is addressed by implementing low pass filters.

Regarding the feed forward controller, there are two different architectures, as presented in Fig. 4 and Fig. 5. The design in Fig. 4 is referred to flatness based feed forward controller, whereas the structure in Fig. 5 is known as exact linearization. Depending on the literature, both are referred to as computed torque control [32]. To reduce the risk of confusion, we will avoid the term computed torque control in this contribution. The flatness based design is explained in detail in Section 3.2 and computes the feed forward torque independently of the current states. It relies exclusively on the reference trajectory and its derivatives and therefore, it can potentially be computed offline. As a consequence, it is invulnerable to any measurement noise. The flatness based architecture is independent of the feedback control and therefore, the feedback control is not time delayed or modified in any sense. It contains the disadvantage of a potentially less precise model since it relies on the reference trajectory rather than the measured trajectory. However, it is possible to partially include online measurements to improve the model accuracy. The inertia matrix, gravity, Coriolis and centripetal terms can be updated at run time based on online measurements.

The exact linearization architecture as presented in Fig. 5, attempts to eliminate the nonlinearity in robot dynamics in an inner loop and therefore control a simple, linear system with the feedback controller. A detailed explanation is given in [13]. Basically, the feedback controller computes an angular acceleration for a dynamic inversion-based controller in order to compensate nonlinear dynamics. Its advantage is that the feedback controller is automatically adjusted to the current robot state. Disadvantages are a necessity of a fast computation of the inverse dynamic model in the inner loop and a potential time delay of the feedback control. In [33] both designs are compared and the flatness based architecture outperforms the exact linearization structure with several feed forward models regarding the achieved trajectory accuracy. The authors in [33] argue that the main advantage 
of a feed forward structure compared to exact linearization is the direct and non-delayed impact of the feedback control law. Based on these arguments, we decide to apply a flatness based architecture rather than exact linearization. The exact linearization module does not account for joint elasticity and therefore, the inner loop reduces to a double integrator. The main idea, to apply the inverse of the model, remains the same in the flatness based feed forward and the exact linearization case. Assume a model inverse of the robot is given by the following form

$$
\tau_{M}=f\left(q_{U}, \dot{q}_{U}, \cdots, q_{U}^{(D)}\right),
$$

with an input angle $q_{U}$ and its derivatives. We define the derivative as $q^{(D)}=\frac{d^{D} q}{d t^{D}}$. In the flatness based architecture, we set $q_{U}=q_{R}$. In the exact linearization formulation, we apply

$$
q_{U}^{(D)}=q_{R}^{(D)}+q_{C}^{(D)},
$$

with the feedback control variable $q_{C}^{(D)}$. With an elastic joint model the derivative order is $D=4$, whereas with the rigid joint model the derivative order is $D=2$ [32]. So, for an elastic joint model we obtain

$$
\ddot{q}_{U}=\ddot{q}_{R}+\ddot{q}_{C},
$$

and for a model with elastic joints we get

$$
q_{U}^{(4)}=q_{R}^{(4)}+q_{C}^{(4)} .
$$

In the exact linearization case it is beneficial to apply the highest input derivative. Note that the output of the velocity controller $u_{C}$ depends on the architecture. In the flatness based case, it is equal to the motor control torque, i.e. $u_{C}=$ $\tau_{C}$. In the exact linearization case, it computes the control input of the inverse dynamics model, i.e. $u_{C}=q_{C}^{(D)}$. In Section 3.2, we derive the flatness based module and thus estimate (12).

\subsection{Feedback Controller}

The enhancement of the feedback controller is a core contribution of this paper. We implement a PD-controller with a link-side position controller using SE and a motorside velocity controller based on the resolver signal. Considering elastic joints it is difficult to precisely calculate the motor reference signals since the equation $\theta_{R}=u q_{R}$ is only valid for rigid joints. A precise calculation of the motor reference signals requires the full, nonlinear stiffness model (1) and (2). Therefore, the exact calculation of the motor reference signals is sensitive to modelling errors. In most previous works, this consideration is simply neglected and the rigid link equation $\theta_{R}=u q_{R}$ is used for the motor-side position and velocity controller. Regarding position control, this problem can be solved by measuring $q$ and implementing a link-side position control. Unfortunately, a link-side velocity control only partially solves this issue. A link velocity speed control utilizes the correct reference speed $\dot{q}_{R}$ but it causes two additional issues. First, a comparative low sensor resolution due to the missing transmission factor requires a significant low pass filtering. Second, to avoid stability issues caused by the elastic joint a soft velocity control parameter is necessary. Both drawbacks can be addressed by implementing a motor-side velocity controller utilizing the full nonlinear elastic joint model for calculating the correct motor reference speed. We present in experiments on the real robot the significance of this effect.

For a compact notation, we define the conventional feedback controller, which neglects joint elasticity as C-FB and the model-based feedback controller, which accounts for joint elasticity as MB-FB. The estimation of the reference velocity $\dot{\theta}_{R}$ model-based feedback controller is presented in Section 3.2. The source code of the implementation is given in Appendix $\mathrm{C}$ and is calculated jointly with the flatness based controller.

Both, the position and velocity controllers are realized as proportional controllers. The position controller is given by

$$
\dot{\theta}_{C}=K_{P}\left(q_{R}-q\right),
$$

with the proportional position gain $K_{P}$ and the motor velocity $\dot{\theta}_{C}$. The velocity controller using the proportional speed gain $K_{V}$ has the form

$$
\tau_{C}=K_{V}\left(\dot{\theta}_{C}+\dot{\theta}_{R}-\dot{\theta}\right),
$$

All in all, the control signal sums up to

$$
u_{v e l}=\tau_{F F}+\tau_{C}
$$

\subsection{Flatness Based Feed Forward Controller}

The main task of the feed forward control law is to achieve a good guiding behavior, whereby the feedback control law can be applied exclusively for the compensation of disturbances and model uncertainties. For the design of the feed forward control law, the inverse nonlinear model of the robot link is used. Therefore, the nonlinear model (1) and (2) is solved for the motor torque $\tau_{M}$, valid for each link, in the form

$$
\tau_{M, i}=J_{i, i} \ddot{\theta}_{i}+\frac{M_{i, i} \ddot{q}_{i}+\tau_{A, i}+\tau_{C C, i}+g_{i}+\tilde{\tau}_{F, i}\left(\dot{q}_{i}\right)}{u_{i}} .
$$

Equation (19) describes the motor torque applied in a specific operating point. We distinguish between variables such as $q_{i}$ and $\theta_{i}$ and parameters such as $M_{i, i}, J_{i, i}, \tau_{A, i}, \tau_{C C, i}$ and $g_{i}$. We neglect all parameter changes during one time interval, in the presented case for $0.8 \mathrm{~ms}$. The parameters are updated each $0.8 \mathrm{~ms}$ according to the state trajectory but are not considered as differentiable variables in the flatness based feed forward control law.

For a good guiding behavior, i.e. $q_{i}=q_{R, i}$, the motor position is set to the motor reference position, i.e. $\theta_{i}=\theta_{R, i}$ and $\tau_{M, i}=\tau_{F F, i}$ is applied. It follows 


$$
\begin{aligned}
\tau_{F F, i} & =J_{i, i} \ddot{\theta}_{R, i} \\
& +\frac{M_{i, i} \ddot{q}_{R, i}+\tau_{A, i}+\tau_{C C, i}+g_{i}+\tilde{\tau}_{F, i}\left(\dot{q}_{R, i}\right)}{u} .
\end{aligned}
$$

In order to solve (20) we need to substitute $\ddot{\theta}_{R, i}$ with a function of $q_{R, i}$ and its derivatives. The following Section shows how to determine each term in (20).

1. The continuous differentiable friction torque $\tilde{\tau}_{F, i}\left(q_{\dot{R}, i}\right)$ is defined in (4).

2. The Coriolis and centripetal torques are summarized for each link in $\tau_{C C, i}$ with

$$
\tau_{C C, i}=\sum_{j=1}^{N} c_{i, j} q_{j}, \quad i \neq j .
$$

Note that $\tau_{C C, i}$ is not a function of $q_{i} . \tau_{C C, i}$ can be calculated using the kinematics of the robot.

3. The link inertia $M_{i, i}$ and the link gravity torque $g_{i}$ can be obtained from the kinematics of the robot. We summarize the inertia torques caused by other links in the acceleration torque $\tau_{A, i}$

$$
\tau_{A, i}=\sum_{j=1}^{N} M_{i, j} \ddot{q}_{R, j}, \quad i \neq j .
$$

4. The motor angle $\theta_{i}$ is substituted in (20) using the inverse nonlinear stiffness model. We use the torsion angle $\Delta q_{i}$ as defined in (5) and solve for $\theta_{i}$

$$
\theta_{i}=u_{i}\left(\Delta q_{i}+q_{i}\right)
$$

The torsion angle $\Delta q_{i}$ is substituted using the inverse, continuously differentiable stiffness model (11) leading to

$$
\theta_{i}=u_{i}\left(\frac{\tilde{\tau}_{E, i}}{c_{T R, i}}+\frac{2 \phi_{B, i}}{1+e^{-s_{E, i}} \tilde{\tau}_{E, i}}-\phi_{B, i}+q_{i}\right) .
$$

The elastic torque is substituted using the sum of torques of the link. Furthermore, we set $\theta_{i}=\theta_{R, i}$ and $q_{i}=$ $q_{R, i}$ and obtain

$$
\begin{aligned}
\theta_{R, i}=u_{i} & \left(\frac{M_{i, i} \ddot{q}_{R, i}+\tau_{A, i}+\tau_{C C, i}+g_{i}+\tilde{\tau}_{F, i}\left(\dot{q}_{R, i}\right)}{c_{T R, i}}\right. \\
& +\frac{2 \phi_{B, i}}{1+e^{-s_{E, i}}\left(M_{i, i} \ddot{q}_{R, i}+\tau_{A, i}+\tau_{C C, i}+g_{i}+\tilde{\tau}_{F, i}\left(\dot{q}_{R, i}\right)\right)} \\
& \left.-\phi_{B, i}+q_{R, i}\right) .
\end{aligned}
$$

Obtaining the second derivative of (25), i.e. $\ddot{\theta}_{R, i}=$ $\frac{d^{2} \theta_{R, i}}{d t^{2}}$, completes the solution for $\tau_{F F, i}$ in (20). MAT$L A B$ Symbolic Math Toolbox was used for solving the equations. It should be pointed out, that due to the precomputation of the solution using $\mathrm{AD}$, the run time of the feed forward calculation requires only a few microseconds. This is only possible because the continuously differentiable formulation of the model allows to use AD tools. The complete source code in $\mathrm{C}$ language is given in Appendix $\mathrm{C}$.

We applied the Robotics Toolbox [34] for the calculation of the inertia matrix $\mathbf{M}(\mathbf{q})$, the gravity load $\mathbf{g}$, the Coriolis and centripetal matrix $\mathbf{C}(\mathbf{q}, \dot{\mathbf{q}})$, as well as for the forward and inverse kinematics. DenavitHartenberg parameters are derived using a CAD model of the robot. We included material densities in the CAD model and utilized the CAD model to calculate mass, inertia, and center of gravity for each link with finite element method (FEM).

\section{Simulation Results}

Before applying the presented algorithm on the $K U K A$ Quantec KR300 Ultra SE, we analyze the feed forward and feedback algorithms in simulation. We compare the flatness based feed forward controller with a nonlinear rigid model feed forward controller. We employ perfect model knowledge, neglect sensor noise and neglect any disturbances in order to focus on pure modelling differences. Unlike on the real robot, we can disable the feedback controller if needed in simulation. We compare the presented algorithm with a nonlinear feed forward control law without elastic joints, i.e. $\boldsymbol{\theta}=\mathbf{U} \mathbf{q}$. As a result, the model in (2) reduces to

$$
\left(\mathbf{M}(\mathbf{q})+\mathbf{J} \mathbf{U}^{2}\right) \ddot{\mathbf{q}}+\mathbf{C}(\mathbf{q}, \dot{\mathbf{q}}) \dot{\mathbf{q}}+\mathbf{g}(\mathbf{q})+\tau_{\mathbf{F}}(\dot{\mathbf{q}})=\mathbf{U} \tau_{\mathbf{M}}
$$

Using (26), the nonlinear feed forward control can be obtained straightforward

$$
\begin{aligned}
\tau_{\mathbf{F F}, \mathbf{R}}= & \mathbf{U}^{-1}\left(\left(\mathbf{M}\left(\mathbf{q}_{R}\right)+\mathbf{J} \mathbf{U}^{2}\right) \ddot{\mathbf{q}}_{R}\right. \\
& \left.+\mathbf{C}\left(\mathbf{q}_{R}, \dot{\mathbf{q}}_{R}\right) \dot{\mathbf{q}}_{R}+\mathbf{g}\left(\mathbf{q}_{R}\right)+\tau_{\mathbf{F}}\left(\dot{\mathbf{q}}_{R}\right)\right) .
\end{aligned}
$$

For an unbiased comparison, we apply the same nonlinear friction (3), Coriolis, centripetal and gravity torque as in the flatness based controller. All model parameters are identical, including the pose-dependent inertia matrix. As a consequence, the only difference between the flatness based and nonlinear rigid model feed forward control law is neglecting the joint elasticity. We independently simulate both feed forward controllers on the same model, (1) and (2). For a compact notation, we define flatness based feed forward control as FB-FF and nonlinear rigid model feed forward control as $\mathrm{R}-\mathrm{FF}$, presented in (20) and (27) respectively.

Fig. 6 presents the reference trajectory, velocity and acceleration used for simulation. For a clear overview, we did not illustrate the jerk and the jerk derivative, although both are continuously differentiable and are used in the FB-FF. For a demanding simulation, we did not choose a standard 


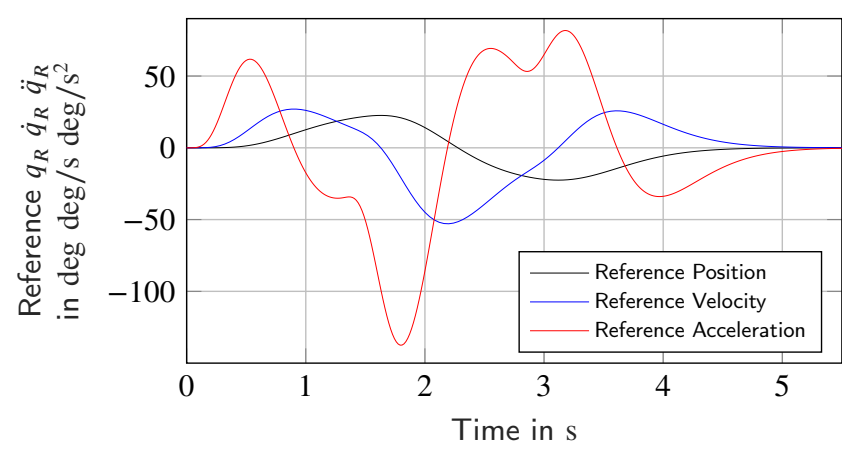

Figure 6: Reference trajectory with angular position, velocity and acceleration used for simulation.

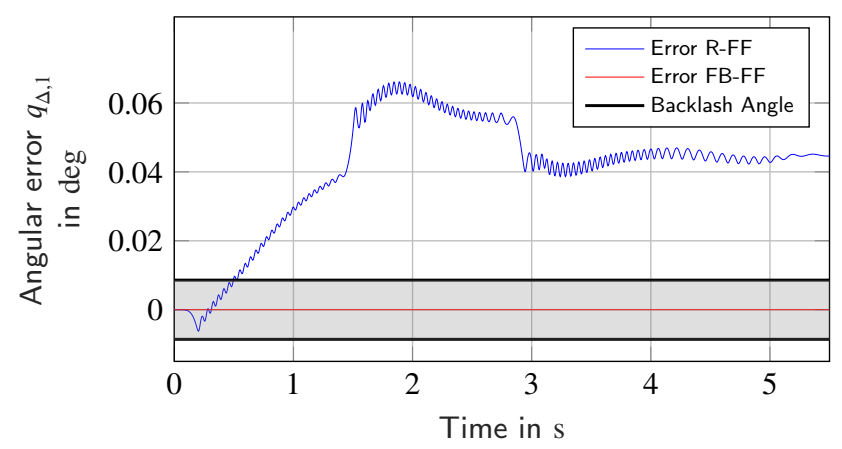

Figure 7: Angular error in simulation with perfect model knowledge, without noise, without feedback controller and without any disturbances. Gray area represents the backlash angle for comparison. FB-FF refers to the flatness based controller and R-FF to the nonlinear rigid model controller.

acceleration and deceleration phase, but rather the trajectory acceleration shown in Fig. 6. In theory, since the simulation does not contain any noise, model errors or external disturbances, the flatness based feed forward controller should perfectly follow an arbitrary trajectory. As presented in Fig. 7, this can be achieved in simulation. Fig. 7 shows the angular error, i.e. $q_{\Delta, i}=q_{R, i}-q_{i}$, for the FB-FF and R-FF in simulation. For comparison, the range of the backlash angle $\phi_{B *}$ is displayed as a gray area in Fig. 7. As expected, the R-FF is slightly worse than the FB-FF. However, a core insight of Fig. 7 is the performance of the R-FF controller on the elastic joint model. Considering the dynamic trajectory, we expected angular errors significantly greater than $0.06 \mathrm{deg}$. Fig. 7 is representative for many simulations confirming this result. If a model error is simulated, i.e. the feed forward model parameters do not match the simulation model parameters, the angular error easily exceeds $>1 \mathrm{deg}$. Note that the simulation presented in Fig. 7 does not apply any feedback control. If an additional feedback controller is applied, which does not account for elastic joints, the angular error increases by an order of magnitude. Therefore, it is very important to implement model based feedback (MBFB) instead of conventional feedback (C-FB) as explained in Section 3.1.

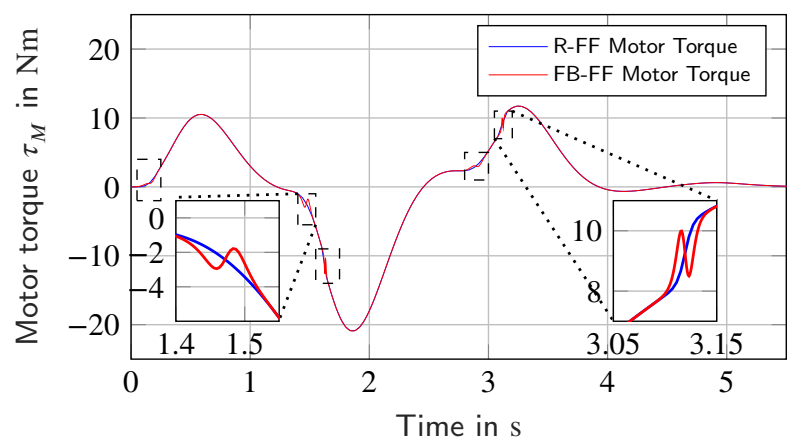

Figure 8: Feed forward motor torque in simulation. FB-FF refers to the flatness based controller and R-FF to the nonlinear rigid model controller.

Fig. 8 compares the feed forward torque of R-FF and FBFF. Due to the identical friction, inertia, gravity, Coriolis and centripetal terms of FB-FF and R-FF, the computed motor torques in Fig. 8 is broadly similar. Differences only occur in the Sections where the model traverses backlash or Coulomb friction. Besides compensating backlash, see Fig. 7, these minor changes in motor torque have huge effects on the elastic torque of the model. As presented in Fig. 9, oscillations induced by backlash within the joint are compensated. Note that we did not employ a SE feedback controller in the simulations, which would be able to damp these oscillations. However, we argue that it is beneficial, if these oscillations are not induced in the first place by a proper flatness based control.

Nevertheless, we identified some detrimental aspects during our analysis in simulation. First of all, modeling backlash as a flat function yields increased motor torque change rates. It is generally known that the less flat a system is, the more dynamic the input variable should be. On a real robot a dynamic input is not desirable. As presented in Fig. 8, the torque change rates can be reduced to a reasonable level for the KUKA Quantec KR300 Ultra SE. However, the demanded torque change rate is the bottleneck of the presented algorithm. This affects the change rate only and the absolute limit of the motor torque was not problematic in any analysis.

Second, backlash is only one source of positional errors. Model errors, sensor noise, external disturbances and conventional feedback controllers have a huge impact on positioning accuracy. Neither, the nonlinear nor the flatness based feed forward torque can account for these effects. For achieving a high positioning accuracy, a model-based feedback controller, ideally with SE, is absolutely necessary.

The parameters used for simulation correspond to joint 1 and are given in Tab. 2.

\section{Experimental Results}

The presented feed forward and feedback control laws are validated on a KUKA Quantec KR300 Ultra SE robot, pictured in Fig. 10. The hardware and robot operating system are based on components from $K U K A$ and industrial supplier $B \& R$ Automation. The $B \& R$ Automation robot op- 


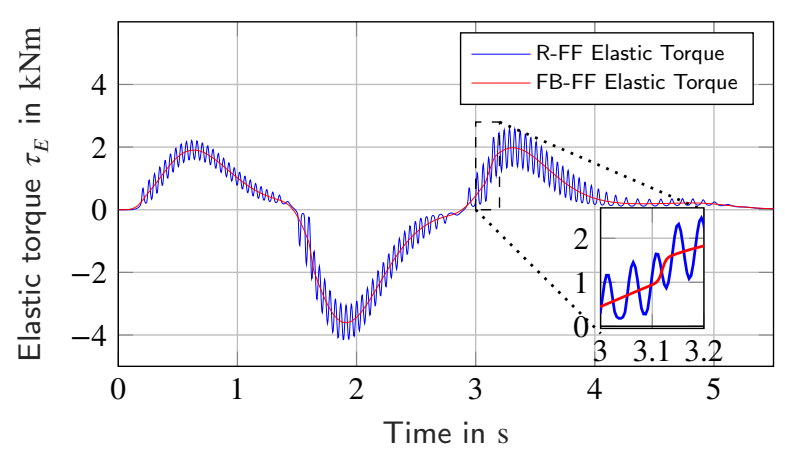

Figure 9: Elastic joint torques in simulation. FB-FF refers to the flatness based controller and R-FF to the nonlinear rigid model controller.

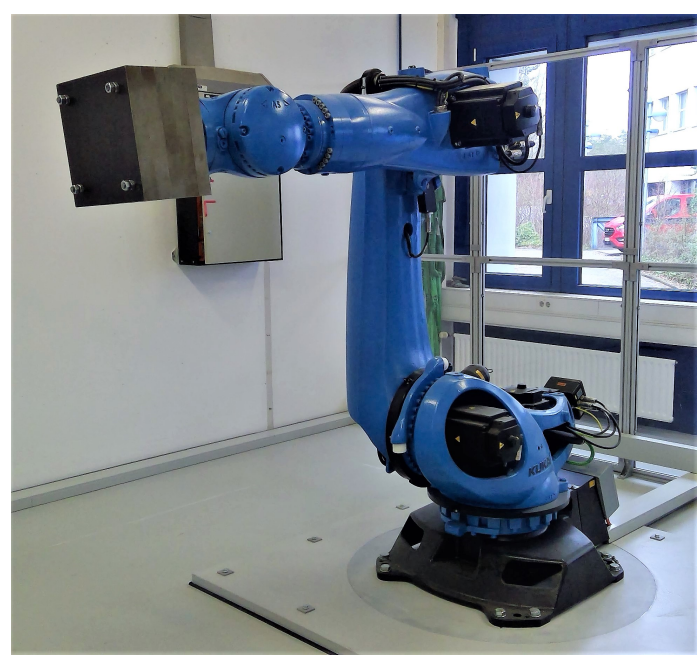

Figure 10: KUKA Quantec KR300 Ultra SE.

erating system allows to implement the control algorithms as presented in Fig. 4. Except for the velocity control which runs in a task class of $0.2 \mathrm{~ms}$, all other presented algorithms run in a task class of $0.8 \mathrm{~ms}$. On the six-joint manipulator, $\mathrm{SE}$ are mounted on the base, shoulder and elbow joints, i.e. joints 1, 2 and 3, and are utilized for position control as illustrated in Fig. 4. The motor encoders of the first three joints are used for velocity control only. Joints 4, 5 and 6 are not equipped with SE and apply motor encoders for position and velocity control. Due to the long lever, we focus our results on the base, shoulder and elbow joints. Due to SE, it is possible to directly measure elastic joint effects and therefore validate the main contributions of this work.

As an experimental scenario, a full robot movement in Cartesian space was chosen. The movement of joints 1,2 and 3 can be measured using the same SE applied for position control. Each SE achieves an accuracy of $\pm 0.017 \mathrm{deg}$. All experiments are carried out on the cold robot, where both gearboxes and motors have approximately a temperature of $24.7^{\circ} \mathrm{C}$, measured before and after the experiments. This leads to a significant effect of nonlinear friction in the base, shoulder and elbow joints. The experiment has been carried out 10 times in a row and the measurements are representa-

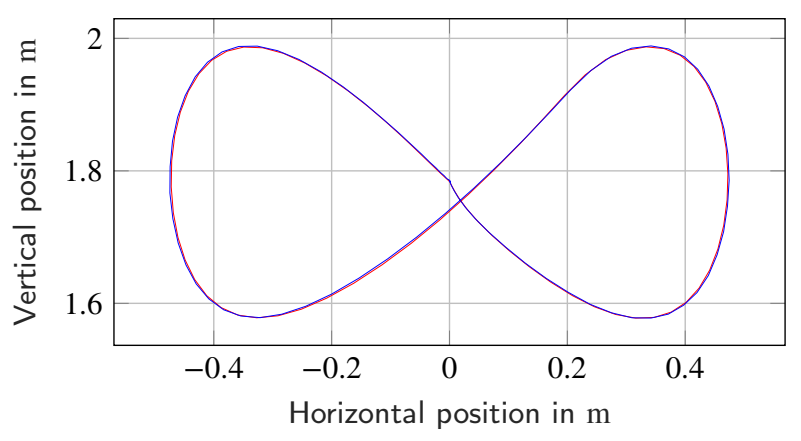

Figure 11: Reference and measured trajectory in Cartesian coordinates of the planar movement. Measurement in blue and reference in red.

tive and reproducible. In order to account for a milling spindle in a robot machining application, and to increase the dynamical loads on each joint, we applied a payload of $150 \mathrm{~kg}$ on the robot's tool center point (TCP) as presented in Fig. 10.

The Cartesian reference trajectory of the movement is shown in Fig. 11. The Cartesian movement starts in a homing position, as presented in Fig. 10, and performs a planar eight-knot movement. The trajectory is based on the Lemniscate of Gerono, which can be parametrized as

$$
\begin{aligned}
& x(t)=a \cos (\varphi(t)), \\
& y(t)=b \sin (\varphi(t)) \cos (\varphi(t))
\end{aligned}
$$

with the horizontal and vertical length parameters $a, b$. The Cartesian angle $\varphi(t)$ performs a $7^{\text {th }}$ order continuously differentiable acceleration and deceleration trajectory. We applied an additional joint space filter after estimating the inverse kinematics. In order to explicitly test effects of backlash, lost-motion and Coulomb friction we chose a Cartesian movement with several changes in direction and parts with link velocities close to zero. The joint space reference trajectory is shown in Fig. 12.

Besides the Cartesian reference trajectory, Fig. 11 also presents the main result: the actual movement of the TCP based on the forward kinematics of the measured link angles. For a detailed discussion, we argue in the following with the measured joint angles and measured motor torques, instead of the estimated Cartesian coordinates with potential errors in the kinematic model.

The angular position improvements are shown in Fig. 13, which present the angular displacement $q_{\Delta, i}=q_{R, i}-q_{i}$ of joint 1,2 and 3 for three cases. We apply the same control parameters, filter constants and dynamic model parameters in all cases. We apply and analyse a movement with a conventional feedback control (C-FB) law as a baseline. The subsequent measurement shows that a model-based feedback controller (MB-FF) leads to an improvement for all joints. A further improvement can be achieved applying the FB-FF and MB-FF. For joints 1, 2 and 3, the maximum path angular error does not exceed $\pm 0.08 \mathrm{deg}$. Note that the angular resolution of the SE is only $\pm 0.017 \mathrm{deg}$. Therefore, the achieved performance of the feedback control law is based only on 5 

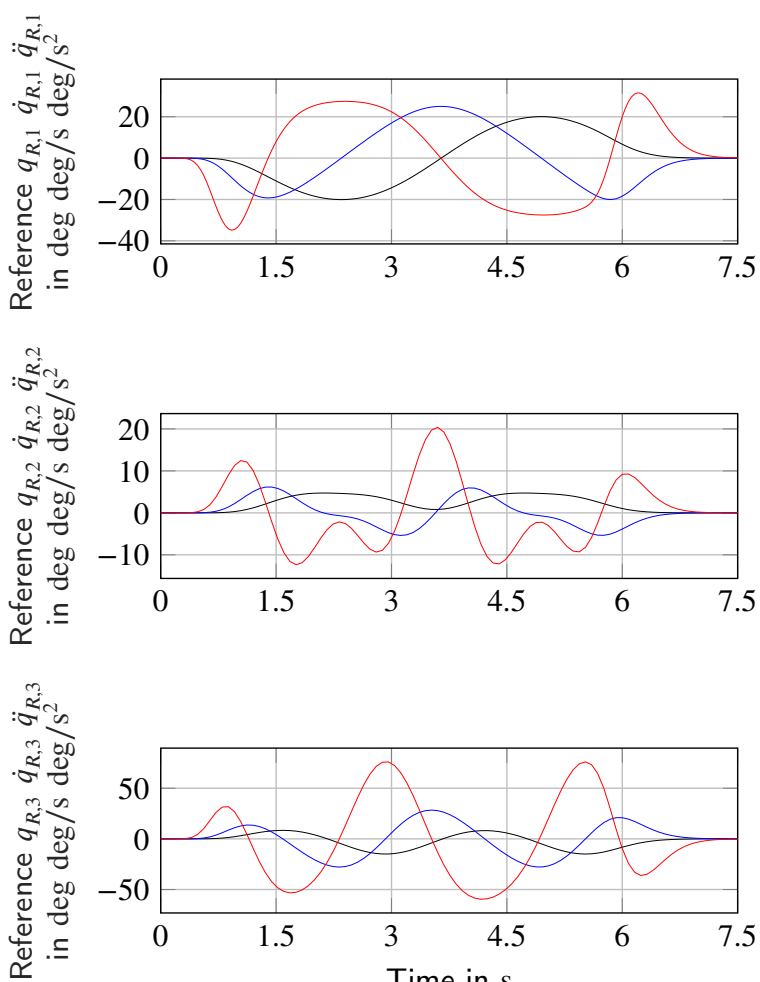

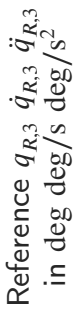

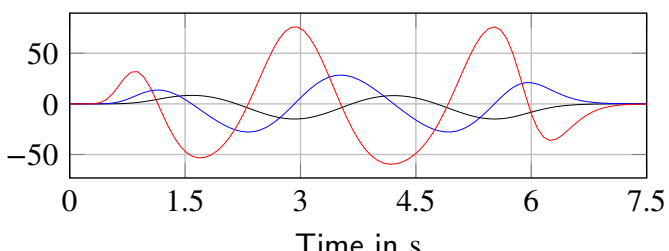

Figure 12: Joint space reference trajectories for each joint. Angular position in black, velocity in blue and acceleration in red.

Table 1

Measured maximum and mean angular errors for all presented algorithms.

\begin{tabular}{lccccc}
\hline algorithm & measure & joint 1 & joint 2 & joint 3 & unit \\
\hline C-FB & max & 0.200 & 0.062 & 0.266 & deg \\
C-FB & mean & 0.087 & 0.020 & 0.106 & deg \\
MB-FB & max & 0.184 & 0.081 & 0.245 & deg \\
MB-FB & mean & 0.081 & 0.019 & 0.101 & deg \\
R-FF & max & 0.135 & 0.053 & 0.080 & deg \\
R-FF & mean & 0.052 & 0.018 & 0.034 & deg \\
FB-FF & max & 0.110 & 0.073 & 0.050 & deg \\
FB-FF & mean & 0.044 & 0.023 & 0.024 & deg \\
\hline
\end{tabular}

measurement increments. All in all, the FB-FF and MB-FB improve the mean error of joint 1 by $49 \%$ and of joint 3 by $77 \%$ compared to C-FB. A detailed analysis of this improvement is presented in Tab. 1 . We refer to mean $\left(\operatorname{abs}\left(q_{\Delta, i}\right)\right)$ as the mean angular error and to $\max \left(\operatorname{abs}\left(q_{\Delta, 1}\right)\right)$ as the maximum angular error in Tab. 1.

As a more comprehensive validation, we compare nonlinear rigid model feed forward control (R-FF with C-FB) and FB-FF with MB-FB on the same trajectory. Modelbased feedback control is not applicable if the rigid joint model neglects elasticity. The experimental result is shown in Fig. 14. The flatness based controller leads to a better performance for joints 1 and 3 than the nonlinear rigid model feed forward control law. The mean error for joint 1 is im-
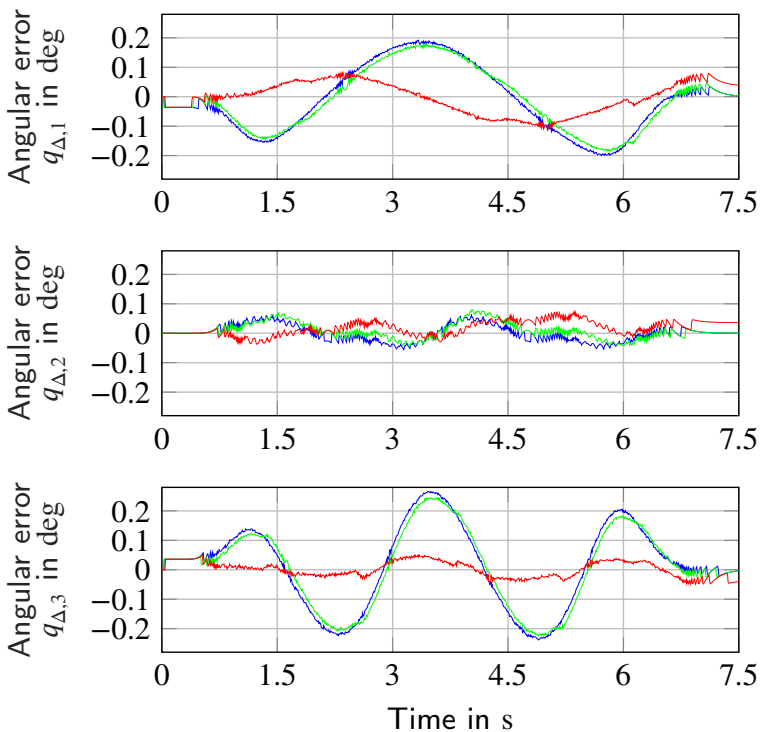

Figure 13: Measured angular error $q_{\Delta, i}$ of each joint. Error with conventional feedback controller ( $\mathrm{C}-\mathrm{FB})$ in blue, model-based feedback controller (MB-FB) in green and flatness based feed forward controller (FB-FF) in red.
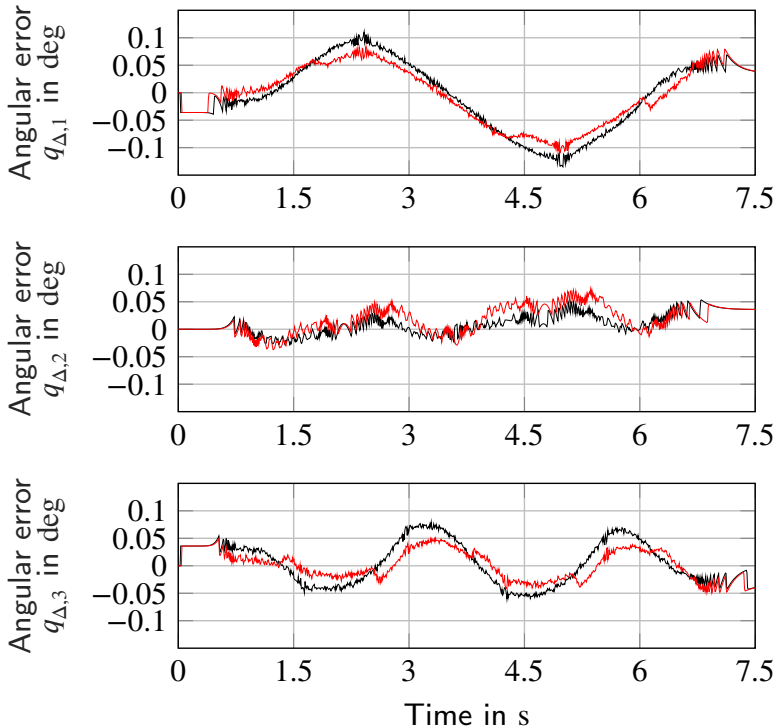

Figure 14: Measured angular error $q_{\Delta, i}$ of each joint. Flatness based feed forward controller (FB-FF) in red and nonlinear rigid model feed forward controller (R-FF) in black.

proved by $15 \%$ and for joint 3 by $29 \%$. The detailed analysis is given in Tab. 1. Regarding joint 2 , both controllers achieve a similar performance of less than $0.073 \mathrm{deg}$ angular error.

Fig. 15 shows the measured motor torque for the proposed flatness based controller including a modified feedback controller for all major axis. For a comparison, we added the pure feed forward torque in the same figure. This measurement demonstrates that the elastic joint model captures the dynamics of joint 1 precisely. Regarding joints 2 and 3 , the measured motor torque significantly differs from 

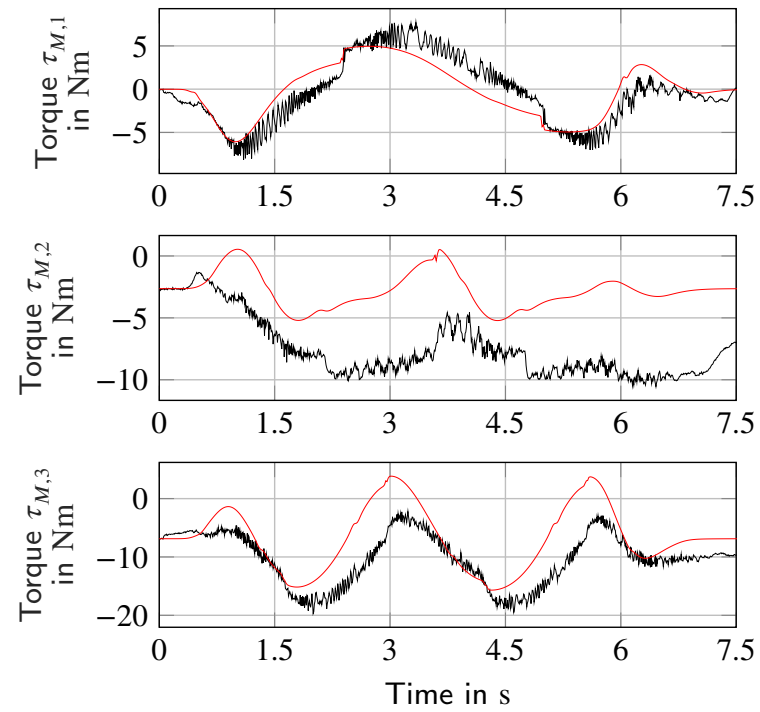

Figure 15: Measured motor torque $\tau_{M, i}$ of each joint in black. The stand-alone flatness based feed forward torque (FB-FF) executed is presented for comparison in red.

the flatness based calculation. Note that the standstill measured motor torques at the beginning of the movement $(0 \mathrm{~s})$ and at its end $(7.5 \mathrm{~s})$ differ significantly. The measured joint angles, see Fig. 13, confirm that the end pose is identical to the start pose within the measurement resolution of $\pm 0.017 \mathrm{deg}$. So, gravity loads and hydraulic spring loads on joint 2 , which only depend on the pose, should be identical at the beginning and at the end. Inertia, Coriolis, centripetal and friction forces are zero in standstill. This leads to the conclusion that a significant asymmetrical friction is present in joint 2 and 3 . For joint 2, the hydraulic counterbalance reduces the gravity torque. Therefore, we neglected both, gravity torque and counterbalance for joint 2 in our model.

In a critical review we find that there is a considerable difference between simulation and real robot results. As remarked before, this is mainly due to model errors, sensor noise and external disturbances which are neglected in the presented simulation. The gap between simulation and experimental results decreases if, for example, model errors are included in the simulation. However, in this case the difference in feed forward torques between FB-FF and R-FF decreases, since both feed forward control algorithms can not account for model errors. Reviewing the feed forward torque comparison, simulation Fig. 8 demonstrates that the nonlinear rigid model feed forward controller (R-FF) already captures the major robot dynamics. A similar result is found in the angular displacement simulation in Fig. 7, where the flatness based controller is better, but the R-FF controller nevertheless achieves an accuracy of $0.06 \mathrm{deg}$. We find this result to be surprising, since the R-FF does not model any joint elasticity. To be clear, the flatness based control is equal or better in all categories. The computed feed forward torque difference is just not significant compared to other error sources like noise, model errors and external distur- bances.

The main advantages of the flatness based controller originate from a different perspective. First of all, note in Fig. 9 the gearbox oscillations are caused by backlash and Coulomb friction. These oscillations feature a considerable amplitude of $1500 \mathrm{Nm}$. Using an accurate flatness based controller eliminates these oscillations.

Second, all in all only the combined performance of feed forward and feedback controller is relevant. It is well-known, that a motor-side velocity controller can achieve a greater bandwidth than a link-side velocity controller. However, for implementing a motor-side velocity controller the calculation of the motor reference velocity $\dot{\theta}_{R, i}$ is non-trivial. Usually, elastic joints are neglected and an incorrect reference is applied, i.e. $\dot{\theta}_{R, i}=u_{i} \dot{q}_{R, i}$. In order to incorporate elastic joint effects, the full nonlinear model dynamics (25) needs to be taken into account. For a rigid joint model, like the nonlinear controller, it is impossible to calculate the correct motor reference velocity since elastic joint effects are neglected in the first place. The flatness based controller is able to fulfil this task and requires only a few microseconds of computation time due to AD. Therefore, the flatness based controller enables a better feedback velocity controller which is a core contribution of this work. We presented in experiments that the proposed algorithm (FB-FF with MB-FB) leads to an improvement of mean accuracy for joint 3 of $29 \%$ compared to R-FF and of $77 \%$ compared to C-FB.

\section{Conclusion}

It was shown that the derived flatness based feed forward controller is capable of utilizing complex, nonlinear dynamical model of the joint flexibility and improving the position precision significantly. Joint backlash and lost-motion can be modeled using a novel nonlinear, continuously differentiable function. This enables standard AD tools, which allow fast run time cycles since the flatness based feed forward controller only requires a few microseconds of computation time online.

During the experiments, we encountered significant difficulties in identifying nonlinear model parameters. Especially the experiments on joints 2 and 3 suggest, that an asymmetrical friction is present. Therefore, future works will further investigate on friction identification for the joints and the hydraulic counterbalance, including Stribeck and asymmetrical friction effects. Our future work will focus on online, nonlinear system identification. Parameter identification during run time enables model adaption to time-varying parameters, such as temperature dependent joint friction. Regarding the vision of robot machining applications, the improvement in trajectory tracking accuracy developed in this contribution enhances industrial robots capabilities for robot machining applications. Future works consider applying and evaluating this algorithm in a robot machining application. 
Table 2

Model parameters of KUKA Quantec KR300 Ultra SE.

\begin{tabular}{|c|c|c|c|c|c|c|c|c|}
\hline description & symbol & joint 1 & joint 2 & joint 3 & joint 4 & joint 5 & joint 6 & unit \\
\hline Coulomb friction & $f_{c}$ & 200 & 150 & 180 & 150 & 150 & 150 & $\mathrm{Nm}$ \\
\hline viscous friction & $f_{v}$ & 800 & 500 & 600 & 100 & 100 & 100 & $\mathrm{Nms} / \mathrm{rad}$ \\
\hline friction smoothness factor & $s_{F}$ & 500 & 300 & 100 & 200 & 200 & 200 & $\mathrm{~s} / \mathrm{rad}$ \\
\hline proportional speed gain & $K_{V}$ & 0.015 & 0.015 & 0.015 & 0.015 & 0.015 & 0.015 & $\mathrm{Nms} / \mathrm{rad}$ \\
\hline proportional position gain & $K_{P}$ & 20 & 20 & 20 & 20 & 20 & 20 & $1 / \mathrm{s}$ \\
\hline backlash angle & $\phi_{B *}$ & 0.15 & 0.15 & 0.15 & 0.15 & 0.15 & 0.15 & $10^{-3} \mathrm{rad}$ \\
\hline lost-motion angle & $\phi_{L M}$ & 0.15 & 0.15 & 0.15 & 0.15 & 0.15 & 0.15 & $10^{-3} \mathrm{rad}$ \\
\hline torsional rigidity stiffness & $c_{T R}$ & 8.4225 & 8.9381 & 5.5691 & 1.6845 & 1.6845 & 1.0726 & $10^{6} \mathrm{Nm} / \mathrm{rad}$ \\
\hline stiffness smoothness factor & $s_{E 2}$ & 0.02 & 0.015 & 0.015 & 0.015 & 0.015 & 0.015 & $1 / \mathrm{Nm}$ \\
\hline gearbox ratio & $u_{G}$ & $1798 / 7$ & $1872 / 7$ & $757 / 3$ & $221 / 1$ & $5032 / 21$ & $206793 / 1340$ & - \\
\hline motor inertia & $J$ & 0.0138 & 0.0177 & 0.0177 & 0.0150 & 0.0150 & 0.0150 & $\mathrm{kgm}^{2}$ \\
\hline
\end{tabular}

\section{Appendix}

\section{A. Robot Model Parameters}

The Tab. 2 shows the parameters utilized of KUKA Quantec Ultra SE.

\section{B. Continously Differentiable Stiffness}

The idea for a continuously differentiable function for the nonlinear stiffness is borrowed from control theory. Consider a PT1-System with a ramp input and set the slope of the ramp equal to the linear stiffness.

$$
T \frac{\mathrm{d} y(t)}{\mathrm{d} t}+y(t)=c_{T R} t
$$

The system output slope will converge towards the input slope, with a constant time offset. Although we are not looking for a time-domain function at all, we can still use the algebraic solution of (29), by replacing the time variable $t$ with the torsion angle $\Delta q$, the output $y(t)$ with the elastic torque $\tau_{E}(\Delta q)$ and setting all initial conditions to zero. In this work, we used a $3^{\text {rd }}$ order system to increase the curvature of the function. Further curvature can be achieved by applying an arbitrary higher order. If the order greater than 1 is applied, two issues have to be considered. First, all poles of the transfer function must coincide. Second, the poles must be adapted to the order $n \in \mathbb{N}^{+}$of the ODE by setting $T=\phi_{B} / n$. This ensures that asymptote of the solution matches the full-contact stiffness. We define the variableorder ODE

$$
\sum_{k=0}^{n}\left(\frac{\phi_{B}}{n}\right)^{k}\left(\begin{array}{l}
n \\
k
\end{array}\right) \frac{\mathrm{d}^{k} y(t)}{\mathrm{d} t^{k}}=c_{F C} t
$$

with the binomial coefficient $\left(\begin{array}{l}n \\ k\end{array}\right)$.

\section{Feed Forward Controller}

We present the flatness based control algorithm, which is obtained by MATLAB Symbolic Toolbox with additional manual modifications and transferred into $\mathrm{C}$. We limited all exponential functions to $\exp (\cdot) \leq 1 e 30$ in order to avoid NotA-Number and infinity errors when using division. We extended the code for estimating all robot joints.

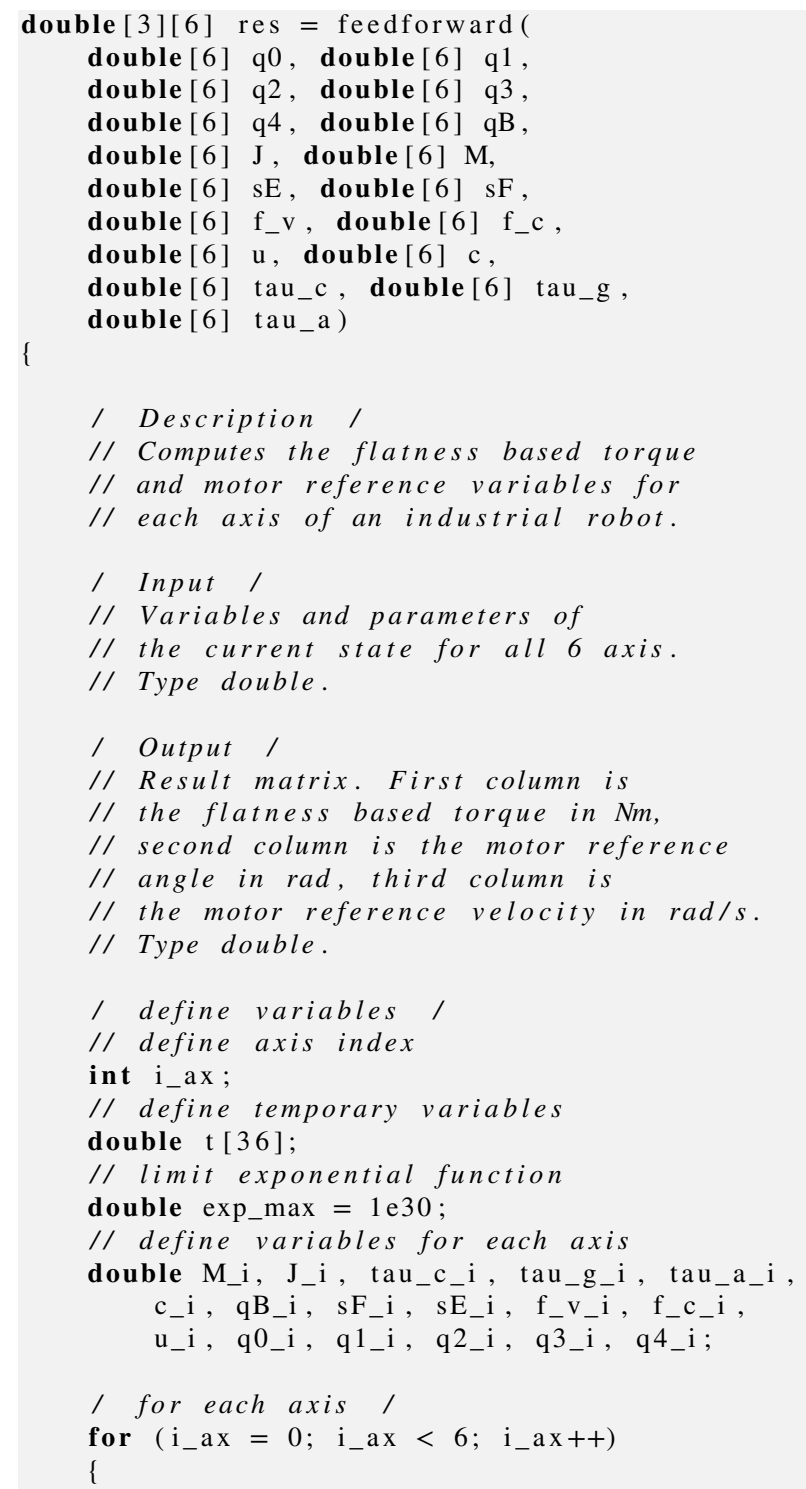




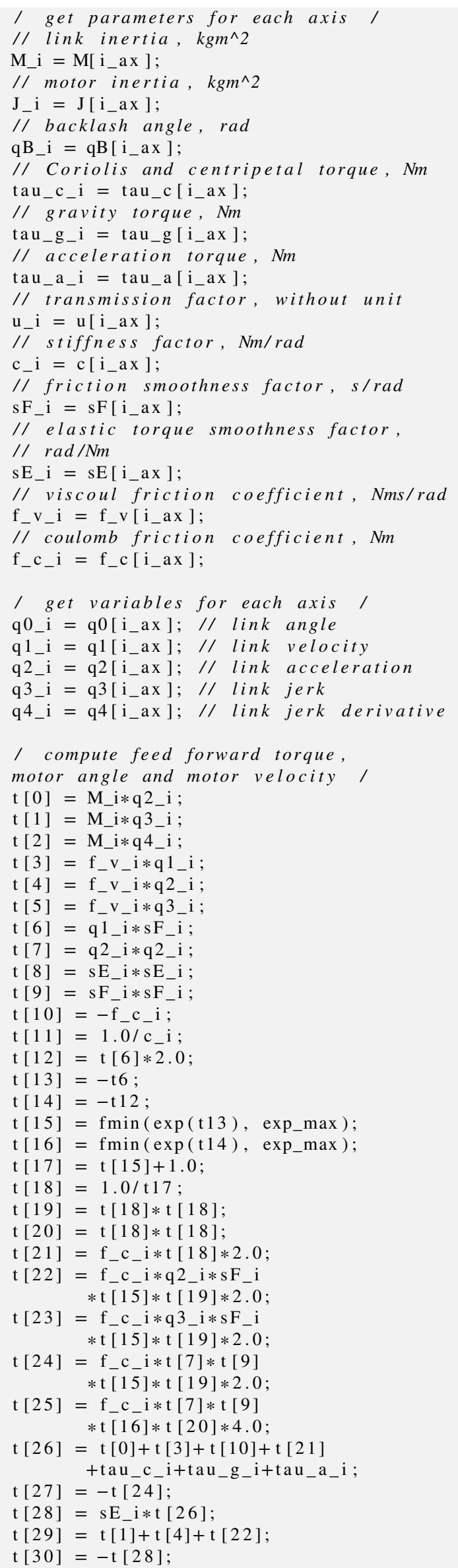

$\mathrm{t}[31]=\mathrm{t}[29] * \mathrm{t}[29]$

\section{References}

[1] A. Olabi, M. Damak, R. Bearee, O. Gibaru, S. Leleu, Improving the accuracy of industrial robots by offline compensation of joints errors, 2012 IEEE International Conference on Industrial Technology, ICIT 2012, Proceedings (2012) 492-497.

[2] M. Freising, S. Kothe, M. Rott, H. Susemihl, W. Hintze, Increasing Accuracy of Industrial Robots in Machining of Carbon Fiber Reinforced Plastics, in: Lecture Notes in Production Engineering, September, 2014, pp. 115-121. doi:10.1007/978-3-319-01964-2\{\\}16.

[3] U. Schneider, M. Momeni-K, M. Ansaloni, A. Verl, Stiffness modeling of industrial robots for deformation compensation in machining, IEEE International Conference on Intelligent Robots and Systems (2014) 4464-4469.

[4] I. Iglesias, M. A. Sebastián, J. E. Ares, Overview of the State of Robotic Machining: Current Situation and Future Potential, Procedia Engineering 132 (2015) 911-917.

[5] K. Wu, C. Krewet, B. Kuhlenkötter, Dynamic performance of industrial robot in corner path with CNC controller, Robotics and Computer-Integrated Manufacturing 54 (2018) 156-161.

[6] A. Brunete, E. Gambao, J. Koskinen, T. Heikkilä, K. B. Kaldestad, I. Tyapin, G. Hovland, D. Surdilovic, M. Hernando, A. Bottero, S. Anton, Hard material small-batch industrial machining robot, Robotics and Computer-Integrated Manufacturing 54 (2018) 185-199.

[7] L. Yuan, Z. Pan, D. Ding, S. Sun, W. Li, A Review on Chatter in Robotic Machining Process Regarding Both Regenerative and Mode Coupling Mechanism, IEEE/ASME Transactions on Mechatronics 23 (2018) 2240-2251.

[8] H. Vieler, A. Karim, A. Lechler, Drive based damping for robots with secondary encoders, Robotics and Computer-Integrated Manufacturing 47 (2017) 117-122.

[9] Y. Lin, H. Zhao, H. Ding, Posture optimization methodology of 6R industrial robots for machining using performance evaluation indexes, Robotics and Computer-Integrated Manufacturing 48 (2017) 59-72.

[10] J. Wang, H. Zhang, T. Fuhlbrigge, Improving machining accuracy with robot deformation compensation, 2009 IEEE/RSJ International 
Conference on Intelligent Robots and Systems, IROS 2009 (2009) 3826-3831.

[11] A. Frommknecht, J. Kuehnle, I. Effenberger, S. Pidan, Multi-sensor measurement system for robotic drilling, Robotics and ComputerIntegrated Manufacturing 47 (2017) 4-10.

[12] R. Devlieg, High-Accuracy Robotic Drilling/Milling of 737 Inboard Flaps, SAE International Journal of Aerospace 4 (2011) 1373-1379.

[13] M. W. Spong, Modeling and control of elastic joint robots, Journal of Dynamic Systems, Measurement and Control, Transactions of the ASME 109 (1987) 310-319.

[14] A. De Luca, P. Lucibello, A general algorithm for dynamic feedback linearization of robots with elastic joints, Proceedings - IEEE International Conference on Robotics and Automation 1 (1998) 504-510.

[15] W. S. Wang, C. H. Liu, Controller Design and Implementation for Industrial Robots with Flexible Joints, IEEE Transactions on Industrial Electronics 39 (1992) 379-391.

[16] A. Albu-Schäffer, C. Ott, G. Hirzinger, A unified passivity-based control framework for position, torque and impedance control of flexible joint robots, International Journal of Robotics Research 26 (2007) 23-39.

[17] P. Mesmer, M. Neubauer, A. Lechler, A. Verl, Drive-Based Vibration Damping Control for Robot Machining, IEEE Robotics and Automation Letters 5 (2020) 564-571.

[18] A. De Luca, W. Book, Robots with Flexible Elements, in: Springer Handbook of Robotics, Springer Berlin Heidelberg, Berlin, Heidelberg, 2008, pp. 287-319. doi:10.1007/978-3-540-30301-5\{\_\}14.

[19] M. Ruderman, F. Hoffmann, T. Bertram, Modeling and identification of elastic robot joints with hysteresis and backlash, IEEE Transactions on Industrial Electronics 56 (2009) 3840-3847.

[20] M. Cordes, W. Hintze, Offline simulation of path deviation due to joint compliance and hysteresis for robot machining, The International Journal of Advanced Manufacturing Technology 90 (2017) 10751083.

[21] J. Brüning, B. Denkena, M. Dittrich, H.-S. Park, Simulation Based Planning of Machining Processes with Industrial Robots, Procedia Manufacturing 6 (2016) 17-24.

[22] T. L. Tran, A. D. Pham, H.-J. Ahn, Lost motion analysis of one stage cycloid reducer considering tolerances, International Journal of Precision Engineering and Manufacturing 17 (2016) 1009-1016.

[23] H. N. Huynh, Edouard Riviere-Lorphevre, O. Verlinden, Multibody modelling of a flexible 6-axis robot dedicated to robotic machining, The 5 th Joint International Conference on Multibody System Dynamics (2018) 1-18.

[24] N. M. Kircanski, A. A. Goldenberg, Experimental study of nonlinear stiffness, hysteresis, and friction effects in robot joints with harmonic drives and torque sensors, International Journal of Robotics Research 16 (1997) 214-239.

[25] T. Yang, S. Yan, Z. Han, Nonlinear model of space manipulator joint considering time-variant stiffness and backlash, Journal of Sound and Vibration 341 (2015) 246-259.

[26] T. Yang, S. Yan, W. Ma, Z. Han, Joint dynamic analysis of space manipulator with planetary gear train transmission, Robotica 34 (2016) 1042-1058.

[27] J. Kim, E. A. Croft, Full-state tracking control for flexible joint robots with singular perturbation techniques, IEEE Transactions on Control Systems Technology 27 (2019) 63-73.

[28] X. Yin, L. Pan, Enhancing trajectory tracking accuracy for industrial robot with robust adaptive control, Robotics and Computer-Integrated Manufacturing 51 (2018) 97-102.

[29] B. Zhang, J. Wu, L. Wang, Z. Yu, Accurate dynamic modeling and control parameters design of an industrial hybrid spray-painting robot, Robotics and Computer-Integrated Manufacturing 63 (2020) 101923.

[30] A. De Luca, W. J. Book, Robots with Flexible Elements, in: Springer Handbook of Robotics, Springer International Publishing, Cham, 2016, pp. 243-282. doi:10.1007/978-3-319-32552-1\{\_\}11.

[31] I. The MathWorks, Symbolic Math Toolbox, 2019. URL: https:// www.mathworks.com/help/symbolic/.

[32] S. Moberg, S. Hanssen, On Feedback Linearization for Robust
Tracking Control of Flexible Joint Robots, volume 41, IFAC, 2008. doi:10.3182/20080706-5-kr-1001.02069.

[33] D. Nguyen-Tuong, M. Seeger, J. Peters, Computed torque control with nonparametric regression models, in: 2008 American Control Conference, IEEE, 2008, pp. 212-217. doi:10.1109/ACC.2008.4586493.

[34] P. Corke, Robotics, Vision \& Control, Springer, 2017. 